\title{
Traduire
}

Une autre perspective sur r tr traduction

Revue française de la traduction

$226 \mid 2012$

Face au miroir

\section{L'interprète au quotidien : littérature, mythe et réalité}

\section{Anne-Marie Cervera}

\section{(2) OpenEdition}

1 Journals

Édition électronique

URL : http://journals.openedition.org/traduire/154

DOI : $10.4000 /$ traduire.154

ISSN : 2272-9992

Éditeur

Société française des traducteurs

\section{Édition imprimée}

Date de publication : 1 janvier 2012

Pagination : 77-81

ISBN : 039-773X

ISSN : 0395-773X

\section{Référence électronique}

Anne-Marie Cervera, «L'interprète au quotidien : littérature, mythe et réalité », Traduire [En ligne], 226 |

2012, mis en ligne le 03 février 2014, consulté le 10 octobre 2020. URL : http://

journals.openedition.org/traduire/154; DOI : https://doi.org/10.4000/traduire.154 


\title{
L'interprète au quotidien : littérature, mythe et réalité
}

\author{
Anne-Marie Cervera
}

1 En entendant parler du thème choisi pour le numéro 226 de Traduire sur l'image du traducteur et de l'interprète dans la littérature, j'ai tout de suite pensé au roman de Javier Marías, Corazón tan Blanco dont le personnage principal, Juan, est interprète pour des organisations internationales.

2 Le sujet m'inspire et j'ai envie d'écrire un article à mon tour. Jusqu'à récemment, je n'aurais jamais osé proposer ma prose à la revue Traduire, dont les articles plutôt théoriques étaient assez éloignés de mes connaissances, de mes intérêts et de ma pratique. Je suis une pragmatique, une personne de terrain, qui n'a jamais suivi de formation classique, incapable de disserter sur les théories de la traduction, mais les derniers numéros de la revue me semblent beaucoup plus accessibles et je pense que mon point de vue sur la question peut intéresser certains.

3 Si je suis une lectrice et une spectatrice assidue et enthousiaste, je n'ai aucun talent de critique littéraire. J'avais commencé à réfléchir à l'article et entamé sa rédaction quand j'ai appris que Christine Cross, à partir de la même idée, avait déjà écrit un article que j'ai lu avec beaucoup d'intérêt - et aussi de soulagement, car sa critique du roman de Javier Marías me convient parfaitement. Elle m'évite en partie cet exercice redoutable consistant à résumer et critiquer un ouvrage, me rappelant mes angoisses de lycéenne les jours précédant la date de remise d'un commentaire de texte, ou pire encore, les séances, souvent dominicales, comme mère d'élèves en rébellion contre cet exercice honni de la «fiche de lecture ", censé donner le goût de la lecture et tant prisé dans l'enseignement du français. Je renvoie donc le lecteur à l'article rédigé par ma collègue pour l'analyse générale du roman, je me contenterai d'ajouter quelques remarques.

4 Corazón tan Blanco, publié par Editorial Anagrama, paraît en Espagne en 1992. Cette œuvre connaît un succès certain et va être traduite dans plusieurs langues. La traduction française Un cœur si blanc, d'Alain et Anne-Marie Keruzoré, publiée par les Éditions Rivages en 1993 est remarquable. Si je lis habituellement la littérature hispanophone dans la version originale, il m'arrive de choisir une traduction, en 
français ou en anglais, et je passe mon temps à détecter les traces du texte original, à deviner quelle pourrait être la version originale. Pour le roman de Javier Marías, j’ai très rapidement oublié qu'il s'agissait d'une traduction. C'est le meilleur signe de qualité dans notre métier : l'interprète ou le traducteur devient invisible. Nous sommes des travailleurs de l'ombre.

5 La traduction anglaise, de Margaret Jull Costa, A Heart so White parait en 1995, chez The Harvill Press. Dans ce cas, le traducteur et l'éditeur n'ont pas eu à avoir des états d'âme pour la traduction du titre, une citation de Shakespeare. J'ai seulement parcouru l'ouvrage et ne saurais me prononcer sur la qualité de la traduction, mais je ne peux pas imaginer qu'elle soit médiocre. Javier Marías a été aussi enseignant de littérature espagnole à Oxford et à Boston, de théorie de la traduction à Oxford et à Madrid, il est lui-même traducteur en espagnol de Sterne, Hardy, Stevenson, Conrad, Nabokov, Faulkner, Salinger...

6 Dans la version française comme dans la version anglaise, on peut remarquer une dédicace des traducteurs - je n'en avais jamais vue auparavant. Pour Un cœur si blanc, en page 8 au verso de la page de dédicace de l'auteur: "Les traducteurs tiennent à remercier Javier Marías de l'aide qu'il leur a apportée. » En haut de la page de titre de $A$ Heart so White: «The translator would like to thank Javier Marías, Annella McDermott and Loreto Todd for all their help and advice. M.J.C. ».

7 L'ouvrage est également traduit en allemand en 1996 par Elke Wehr, sous le titre Mein Herz so weiß. Le roman connaît un énorme succès en Allemagne, avec plus d'un million d'exemplaires vendus à ce jour. Ce qui a attiré mon attention en feuilletant cette version, c'est le résumé d'un des éditeurs : « Le narrateur est interprète et souffre d'une déformation professionnelle; il ne peut pas s'empêcher d'enregistrer et d'interpréter tous les détails. » Voilà une description parfaite de l'intérêt tout particulier du roman pour nous les maniaques des langues souffrant parfois de TOC (traduction obsessionnelle compulsive).

8 Tout au long du roman, le héros Juan parle de langues, de traduction, d'écoute, en espagnol, anglais et français. Je ne vais pas me lancer dans les citations, je vous laisse le plaisir de découvrir le texte par vous-mêmes, dans la langue de votre choix. Voyons maintenant la description de la vie d'un interprète au quotidien.

10 Juan, le héros interprète, traverse une période personnelle difficile; alors que tout semble aller pour le mieux dans sa vie, il éprouve un malaise constant, le sentiment que le malheur est imminent. Dans cet état d'esprit, il ne voit plus que le côté obscur, le verre à moitié vide. Un chapitre entier est consacré à la description de son activité (page 57 à 71 pour la version française). Pratiquement toutes les remarques concernant la vie professionnelle du héros tout au long du livre portent sur les difficultés qu'engendre l'exercice de la profession, en particulier à Genève et à New York. Il faut attendre la page 219 de la version française pour entendre un commentaire plus positif de Juan : «Ce n'est pas que ce métier ne m'intéresse pas énormément, mais dans cette ville, et en hiver, il me devenait insupportable ». J'ai recherché la traduction anglaise de cette phrase, et j'ai été surprise de lire : «It's not just that I've never found the work interesting, but in that particular city, in winter, I found it unbearable ». Ce n'est pas du tout la même chose, je vais donc rechercher le texte original: « No es que nunca me haya interesado mucho el trabajo, pero en aquella ciudad, y en invierno, se me hacía insoportable». 
11 Je n'ai absolument pas l'intention de jeter la pierre à des collègues dont le travail globalement est excellent, je souhaite simplement souligner la difficulté de notre tâche, et l'impossibilité de faire un sans-faute dans un très long texte - c'est aussi une manière de me rassurer, je ne suis pas la seule à laisser des imprécisions, cela arrive même aux meilleurs.

12 Le terme officiel pour désigner le métier qui nous occupe est celui d'interprète de conférence. L'interprétation de conférence "pure et dure " est pratiquée pour des colloques, conférences, symposiums, congrès, séminaires, conventions... $\mathrm{Ne}$ me demandez pas les différences entre ces termes, je ne les ai toujours pas comprises après des années de pratique, je les entends utilisés indifféremment pour désigner le même type d'événement (on peut aussi parler de grand-messes, dans un autre registre). Les interprètes sont dans une cabine isolée, n'ont pas de contacts avec ceux qui les écoutent, ou alors rarement, pendant les pauses. Dans la réa- lité, les conférences ne représentent qu'une partie, plus ou moins importante, dans le travail de nombreux interprètes de conférence. Il y a des réunions : certaines sont formelles et impliquent souvent un nombre élevé de participants, avec des cabines pour les interprètes; d'autres sont plus informelles et, dans ce cas, les interprètes ne sont plus dans leur bulle isolée, mais dans la salle même de la réunion, munis d'un micro haute fréquence sans fil dans lequel ils chuchotent le plus discrètement possible la traduction, un «bidule » dans notre jargon. Dans ces réunions, il arrive souvent que les intervenants ne disposent pas d'un micro, le son qui par- vient aux oreilles des interprètes est donc beaucoup moins clair. Les participants peuvent prendre la parole à tout moment, parfois plusieurs personnes s'expriment en même temps quand les esprits s'échauffent, tout dépend de la discipline qui règne dans le groupe, de la présence ou non d'un modérateur ou d'un président de séance. Les réunions sont très variées : conseil d'administration d'association ou de société, planification d'un événement, comité d'entreprise, négociations autour d'un plan social, réunion financière, remueméninges, la liste est longue. Certains entretiens sont également interprétés, pour une embauche, un licenciement, une étude de marché. Notre présence peut être requise pour des visites d'usine, de laboratoire, des formations... L'activité est très variée et à mon avis, le terme d'interprétation simultanée conviendrait mieux. À propos de ce mot, interprétation, un détail m'a amusée dans la traduction française. Dans la profession, il existe un grand débat, souvent passionné, sur l'utilisation du mot "interprétariat ", proscrit par certains pour des raisons que je ne comprends pas toujours très bien. Quoiqu'il en soit, c'est le terme interprétariat qui est utilisé dans le roman.

13 Le métier peut se pratiquer dans des organisations internationales (ONU, OMS, Commission européenne, Cour de Justice européenne, Tribunal pénal international, etc.), avec un statut de fonctionnaire, ou en tant que freelance, comme Juan. Le statut de freelance n'est pas toujours un choix, pour certains il s'agit d'une étape dans le processus qui va les mener au statut de fonctionnaire, en attendant qu'un concours soit ouvert, qu'une place se libère, mais pour d'autres, c'est une volonté d'être libre dans leurs choix de travail et de vie en général.

Le métier s'exerce aussi sur le marché privé, par l'intermédiaire d'agences ou pour des clients directs. Il existe ce qu'on appelle un marché « gris »; ce sont des missions moins bien payées, avec des conditions de confort, de qualité d'écoute, d'équipement, d'horaires de travail, moins favorables. Et en bas de l'échelle un marché «noir », mais contrairement à ce que le terme pourrait laisser croire, vous n'allez pas être payé en 
cash et échapper à l'imposition. Non, la "défiscalisation » est réservée, en toute légalité, aux interprètes accrédités auprès de certaines organisations internationales. $\mathrm{Si}$ ce marché est noir, c'est que les conditions de travail vont être nettement plus défavorables. Certains freelances exercent à la fois sur le marché privé et au sein d'organisations internationales.

Souvent, dans le milieu des interprètes, le travail au sein des institutions est considéré comme la voie royale, celle que choisiront les meilleurs, celle où on retrouvera l'élite de la profession et il existe une hiérarchie de fait, avec au sommet les collègues fonctionnaires d'une institution internationale.

Ces différents degrés de prestige et de respectabilité au sein de la profession me déplaisent. Nous ne sommes pas tous bons pour les mêmes choses, certains seront excellents dans des situations très formelles, pour la traduction de discours complexes mais seront incapables de s'adapter à une réunion qui partira un peu dans tous les sens, au cours de laquelle les esprits vont s'échauffer (plan social, fermeture de site, par exemple) mais pour laquelle il est particulièrement important que toute la colère et la frustration que veulent exprimer les participants, y compris avec des noms d'oiseaux, soient communiquées dans une autre langue, parfois en termes violents. Un interprète excellent pour suivre des réunions de consommateurs dans une étude de marché pourra ne pas savoir traduire dans toute sa subtilité la langue de bois d'un discours politique. Nos talents sont divers et chacun doit trouver la place qui lui convient et le respect qu'il mérite.

Je vais faire une comparaison avec les restaurants pour illustrer mon propos. Selon l'humeur, les commensaux impliqués, l'heure du repas, l'appétit, le temps disponible, l'endroit où je me trouve, l'état des finances - les miennes ou celles de celui qui se chargera de l'addition - je vais aller dans un gastro, un fast-food, un resto de quartier avec un seul et unique "pladuj ", un bouchon, un semi-gastro, un self, un étoilé. Les serveurs et cuisiniers impliqués dans mon expérience de repas auront des aptitudes et qualités très diverses. Vivacité, rapidité, sympa- thie pour le serveur du resto de quartier, patience, élégance pour celui d'un gastro; minutie dans l'élaboration de préparations très complexes pour le cuisinier d'un étoilé, rapidité et organisation minutée pour une courte et nourrissante pause de midi. Mais dans tous les cas, je veux de la qualité chez des professionnels, qui bénéficieront tous de ma considération.

Vous pensez peut-être que je suis en train de mélanger torchons et serviettes, mais il me semble utile de démystifier notre travail pour mieux défendre tous ceux qui l'exercent. Le côté un peu glamour de la vie menée par « l'élite » de notre profession est bien mis à mal dans le roman de Javier Marías.

L'interprète au quotidien - vie de rêve, mythe ou réalité ? 

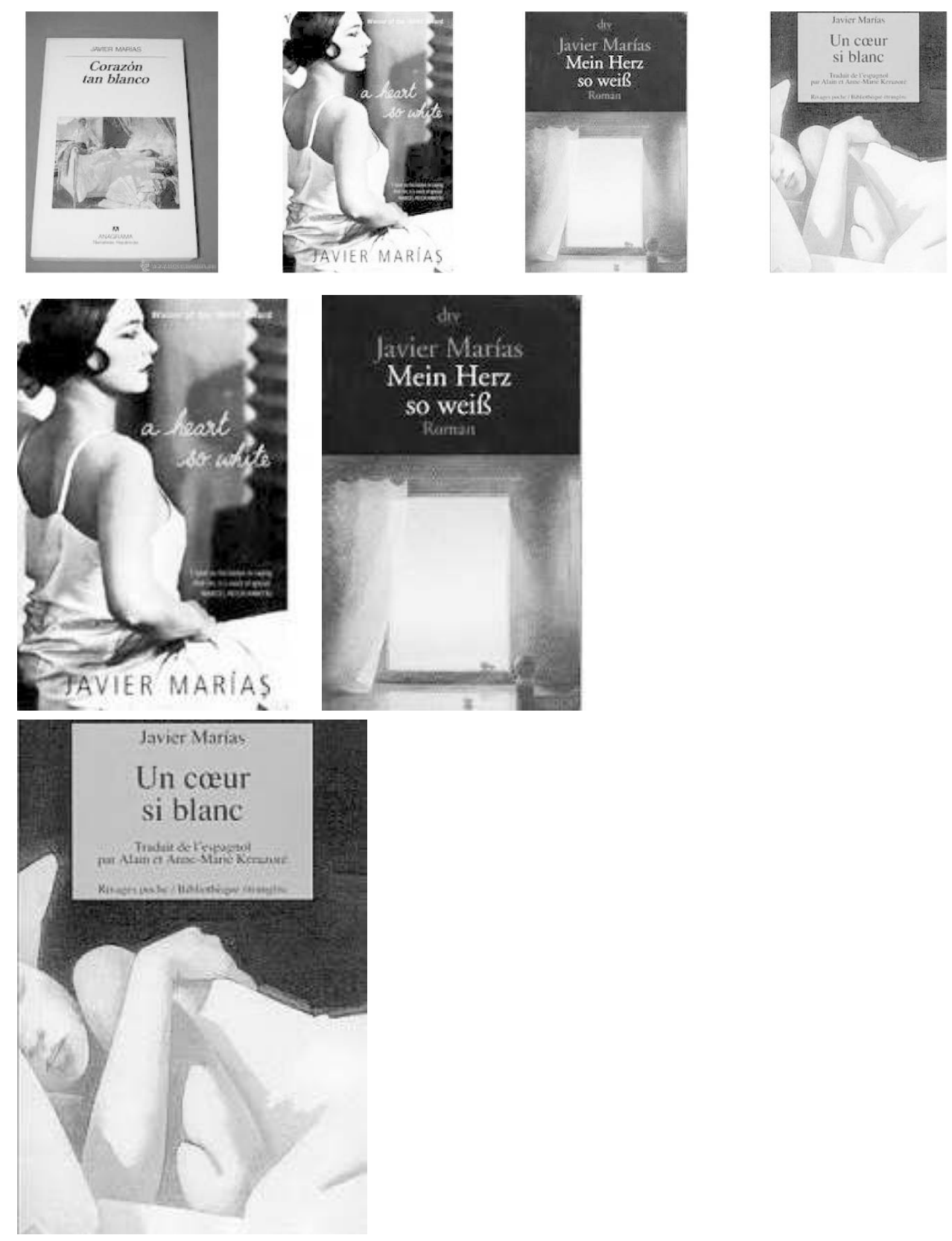

\section{BIBLIOGRAPHIE}

Javier MARÍAS, Corazón tan blanco, Editorial Anagrama, 1992.

Un cœur si blanc, traduit de l'espagnol par Alain et Anne-Marie Keruzoré, éditions Rivages, 1993.

A Heart so White, translated by Margaret Jull Costa, The Harvill Press, 1996.

Mein Herz so weiß, aus dem Spanischen von Elke Wehr, J.-G. Cotta'sche Buchhandlung, 1996. 
AUTEUR

ANNE-MARIE CERVERA

annemarie.cervera@gmail.com 